\title{
Neumolaberinto como complicación de otitis media crónica colesteatomatosa
}

\author{
Pneumolabyrinth as a complication of chronic otitis media with cholesteatoma
}

\author{
Michelle Lupa Mendlovic, ${ }^{*}$ Francisco Miguel Fernández Andrade, ${ }^{*}$ \\ Ramón Amayo Ramírez, ${ }^{\ddagger}$ Juan Carlos Cisneros Lesser ${ }^{\ddagger}$
}

\begin{abstract}
RESUMEN
La fístula perilinfática se define como una comunicación anómala entre el oído interno y el oído medio; tanto el sistema auditivo como vestibular pueden estar afectados en diferentes grados. Los síntomas principales son hipoacusia neurosensorial progresiva y vértigo con esfuerzos. El término «neumolaberinto» hace referencia a la presencia de aire dentro del laberinto. Suele ser un hallazgo infrecuente que confirma el diagnóstico de una fístula perilinfática; la causa más frecuente es la traumática. En el contexto de la otitis media crónica con colesteatoma, es común la presencia de fístula laberíntica por erosión ósea de las estructuras del oído interno. En la fístula laberíntica, a diferencia de la perilinfática, el endostio se mantiene íntegro previniendo la salida de perilinfa. La fístula perilinfática y el neumolaberinto no son complicaciones frecuentes de otitis media crónica colesteatomatosa. El manejo de la fístula perilinfática no está estandarizado, ya que depende de la etiología y la evolución clínica. En la mayoría de los casos, se recomienda un manejo conservador, reservando el tratamiento quirúrgico para casos donde haya sintomatología vestibular severa, progresión de la hipoacusia o sospecha de fístula activa. Presentamos el caso de un paciente de 73 años con otitis media crónica con colesteatoma, quien, posterior a la limpieza en el consultorio, presentó sintomatología compatible con fístula perilinfática, corroborada por neumolaberinto en la tomografía computarizada, secundaria a la erosión del estribo en su totalidad y exposición de la ventana oval. En este caso, consideramos necesario el manejo quirúrgico para erradicar la
\end{abstract}

\begin{abstract}
A perilymphatic fistula is defined as an abnormal communication between the inner ear and middle ear. The auditory and vestibular systems can be affected in different degrees, the main symptoms are progressive sensorineural hearing loss and vertigo. The term "pneumolabyrinth" refers to the presence of air inside the labyrinth, it is an infrequent finding that confirms the diagnosis of a perilymphatic fistula. The most frequent cause is traumatic, other causes are: barotrauma, iatrogenic, erosive and idiopathic. In the context of cholesteatoma, labyrinthine fistula secondary to bone erosion is common. In this, unlike the perilymphatic fistula, the endosteum remains intact. Perilymphatic fistula is not frequently reported as a complication of chronic cholesteatomatous otitis media. The management of perilymphatic fistula is not standardized since it depends on the etiology and clinical evolution of each patient. In most cases, conservative management is recommended, reserving surgical treatment in cases of severe vestibular symptoms, progression of hearing loss or suspicion of active perilymphatic fistula. We present a clinical case of a 73-yearold patient with a history of chronic cholesteatomatous otitis media, who after cleaning and aspiration of otorrhea in the clinic presented symptoms compatible with perilymphatic fistula corroborated by pneumolabyrinth, with erosion of the oval window. pneumolabyrinth is a rare complication of chronic cholesteatomatous otitis media. In this case we consider surgical management was necessary to eradicate the
\end{abstract}

\footnotetext{
* Médico Residente.

¥ Médico adscrito.
}

Otorrinolaringología y Cirugía de Cabeza y Cuello, Instituto Nacional de Rehabilitación (INR), Ciudad de México.

Recibido para publicación: 07/07/2019. Aceptado: 15/11/2019.

Correspondencia: Michelle Lupa Mendlovic

Calzada México-Xochimilco Núm. 289, Col. Arenal de Guadalupe, 14389,

Alcaldía Tlalpan, Ciudad de México. Teléfono: 59991000, ext. 18274

E-mail: michelle.lupa@gmail.com

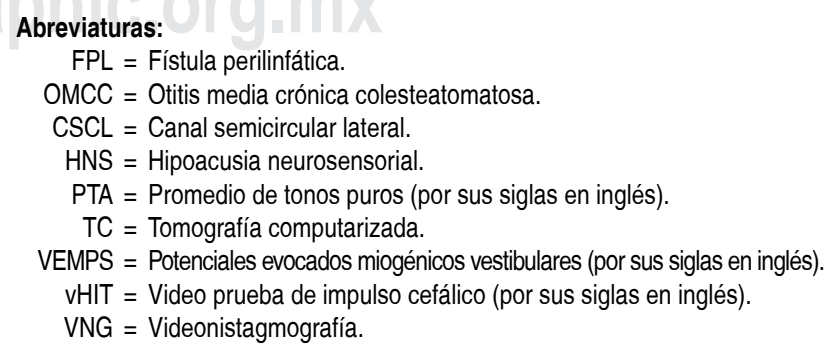

Este artículo puede ser consultado en versión completa en: www.medigraphic.com/analesmedicos 
enfermedad y el cierre de la fístula, disminuyendo el riesgo de complicaciones en el sistema nervioso central.

Palabras clave: Fístula perilinfática, neumolaberinto, colesteatoma, otitis media crónica.

Nivel de evidencia: IV

\section{INTRODUCCIÓN}

La fístula perilinfática (FPL) se define como una comunicación anómala entre el oído interno y el oído medio con salida de perilinfa. Ésta puede ocurrir a través de la ventana oval, de la ventana redonda o por dehiscencias en la cápsula ótica. ${ }^{1}$

La fístula laberíntica - a diferencia de la FPLimplica la erosión ósea sin involucro del endostio, dejando una barrera entre el oído medio y el espacio perilinfático. Ésta tiene una incidencia de 5 a $10 \%$ en casos de otitis media crónica colesteatomatosa (OMCC); el sitio de erosión más común es el canal semicircular lateral (CSCL) en 60 a 80\%., ${ }^{2,3}$ Dornhoffer y Milewski la clasifican en tres tipos, a saber, I: laberinto membranoso recubierto por una capa ósea adelgazada (línea azul); II: membrana perilinfática expuesta, y III: erosión de la membrana perilinfática o presencia de tejido de colesteatoma dentro del laberinto. ${ }^{4}$

El término «neumolaberinto» fue introducido por Mafee en 1984 como la presencia de aire dentro del laberinto. ${ }^{5}$ Es un hallazgo radiológico poco frecuente, que confirma la presencia de FPL. ${ }^{6}$ En 1978, Grundfast y Bluestone fueron los primeros en reportar un caso en el cual identificaron burbujas de aire atrapadas detrás del nicho de la ventana redonda. ${ }^{7}$ La causa más reportada con mayor frecuencia es la traumática por fractura del hueso temporal. Aunque se han reportado otras causas como barotrauma iatrogénica, erosiva e idiopática. ${ }^{1}$

El sistema auditivo y el vestibular pueden afectarse en diferentes grados. El síntoma más frecuente es la hipoacusia neurosensorial (HNS), la cual puede ser fluctuante o persistente. Puede ocurrir hipoacusia conductiva debido a la presencia de aire en el oído interno que limita la movilidad del estribo. Otras manifestaciones son vértigo y mareo, fenómeno de Tullio, acúfeno y plenitud aural. ${ }^{1,5}$

Para su diagnóstico y plan terapéutico se requiere de la tomografía computarizada (TC) de oído y de audiometría tonal. ${ }^{5}$ Los potenciales evocados vestibulares miogénicos (vestibular evoked myogenic potential disease and closure of the fistula, to prevent complications to the central nervous system.

Keywords: Perilymphatic fistula, pneumolabyrinth, cholesteatoma, chronic otitis media.

Level of evidence: $I V$

o VEMPS) cervicales u oculares pueden revelar una disminución en el umbral de activación en el oído afectado. El manejo no está estandarizado, ya que éste depende de la etiología y la evolución clínica de cada paciente. En la mayoría de los casos, la fístula puede resolverse de manera espontánea y el neumolaberinto suele reabsorberse igualmente, ${ }^{5}$ por lo que se recomiendan medidas conservadoras como reposo absoluto, elevación de la cabeza y evitar la maniobra de Valsalva. ${ }^{6}$ El uso de esteroide sistémico, profilaxis antibiótica, vacunación contra $S$. pneumoniae o meningococo y el uso de antivertiginosos son controversiales, aunque generalmente recomendados.

Se indica tratamiento quirúrgico en casos de sintomatología vestibular severa, progresión de la hipoacusia o sospecha de FPL activa. ${ }^{8}$ El pronóstico para los síntomas vestibulares suele ser bueno. En cuanto a la audición, el pronóstico es menos predecible. La hipoacusia parece tener mayor relación con la extensión del daño que con la terapéutica empleada. ${ }^{1}$ El presente artículo reporta el caso clínico de un neumolaberinto secundario a la erosión del vestíbulo como complicación de OMCC, etiología poco reportada en la literatura.

\section{CASO CLÍNICO}

Se trata de un hombre de 73 años de edad con antecedente de mastoidectomía del muro bajo izquierdo por OMCC de 30 años atrás, cuyo seguimiento fue malo. Acudió a valoración clínica por mareo e inestabilidad de dos meses de evolución.

A la exploración física (EF), se halló el oído izquierdo con cavidad de mastoidectomía inactiva y el oído derecho con otorrea y presencia de tejido de queratina proveniente de perforación timpánica de cuadrantes posteriores.

Se realizó limpieza parcial en el consultorio, debido a que el paciente presentó vértigo intenso a la manipulación, el cual cedió con difenidol intravenoso, en una única dosis. A las 24 horas presentó exacerbación del vértigo caracterizado por episodios de 40 minutos de duración, desequilibrio con lateropulsión 


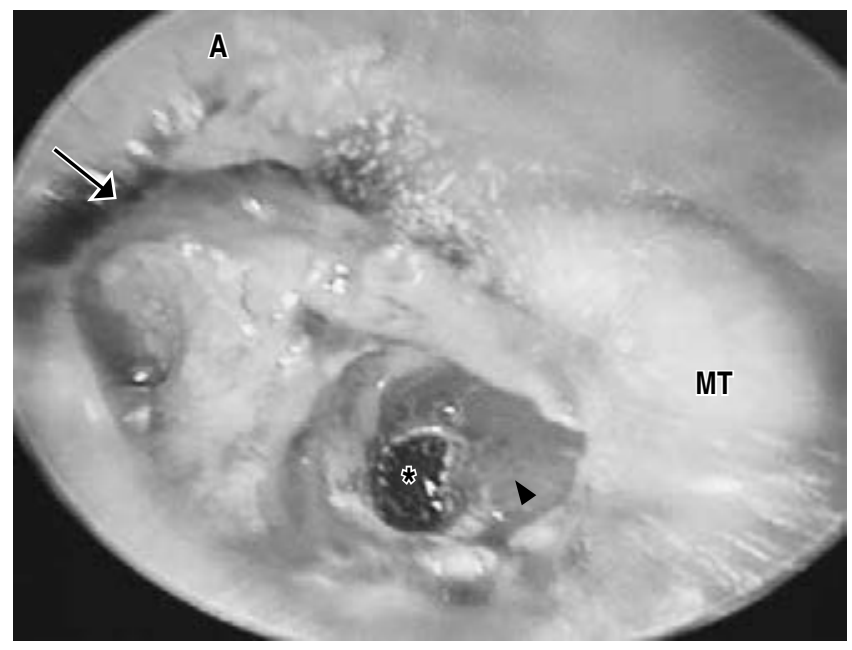

Figura 1: Endoscopia $0^{\circ}$ de oído derecho. Se evidencia erosión de la pared posterosuperior (flecha), ático $(\boldsymbol{A})$, perforación timpánica de cuadrante posterosuperior (cabeza de flecha), ventana oval abierta ( ${ }^{*}$ ), remanente timpánico (MT) con ocupación retrotimpánica.

derecha, náusea, vómito y pérdida auditiva derecha de presentación súbita.

Se encontraron signos vitales dentro de los parámetros normales; sin nistagmo espontáneo o evocado, prueba de impulso cefálico positiva con tracción cefálica derecha. Las marchas se consideraron no valorables por la inestabilidad. Nuevamente, se limpiaron los restos de queratina y otorrea del oído derecho y se observó: erosión de la pared posterosuperior del conducto auditivo externo, perforación timpánica de cuadrantes posteriores con erosión de martillo y yunque, y ausencia de estribo y la ventana oval abierta (Figura 1). En la acumetría, el oído derecho no presentó captación para ninguna de las frecuencias evaluadas en la prueba de Rinne y Weber al oído contralateral.

Se realizó una audiometría tonal que mostraba el oído derecho con hipoacusia neurosensorial profunda (PTA $111 \mathrm{~dB}$ ) y el oído izquierdo con hipoacusia mixta profunda de perfil descendente (reserva $63 \mathrm{~dB}$, PTA $100 \mathrm{~dB}$ ). Se solicitó una tomografía computarizada, donde se observó: ocupación del oído medio, dehiscencia de tegmen, erosión de la cadena osicular, platina ausente y neumolaberinto con extensión hacia el vestíbulo, canales semicirculares y cóclea $(F i$ guras 2 y 3). Se corroboró el diagnóstico de OMCC complicada con FPL y laberintitis bacteriana.

Se decidió iniciar con un manejo conservador mediante medida antifístula, administración de difenidol, esteroide sistémico y antibiótico intravenoso (ceftria- xona $2 \mathrm{~g}$ /día). El paciente se mantuvo afebril, sin datos de infección neurológica y con mejoría de la sintomatología vestibular. Se realizó una videonistagmografía (VNG) y video prueba de impulso cefálico (video head impulse test o vHIT), concluyendo que había disfunción vestibular bilateral de predominio derecho. En una segunda tomografía realizada a los cinco días persistía el neumolaberinto con mismas características. A los 10 días del inicio del cuadro, se le realizó mastoidectomía radical modificada derecha y obliteración de la ventana oval con tejido conectivo. Recibió 10 sesiones de rehabilitación vestibular con mejoría significativa; en la actualidad está sin riesgo de caída. En audiometrías subsecuentes no presentó ganancia auditiva. Se adaptó un auxiliar auditivo izquierdo con buena ganancia y utilidad.

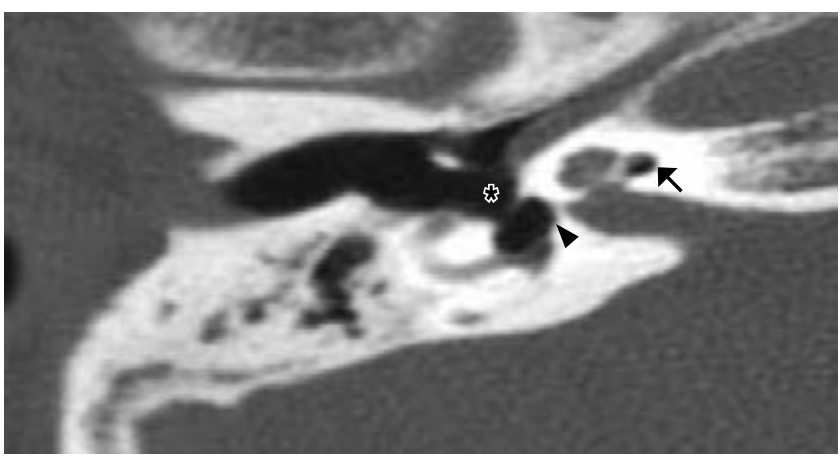

Figura 2: Tomografía computarizada de oído derecho corte axial. Platina ausente (*), neumolaberinto con extensión hacia el vestíbulo (cabeza de flecha) y hacia la cóclea (flecha).

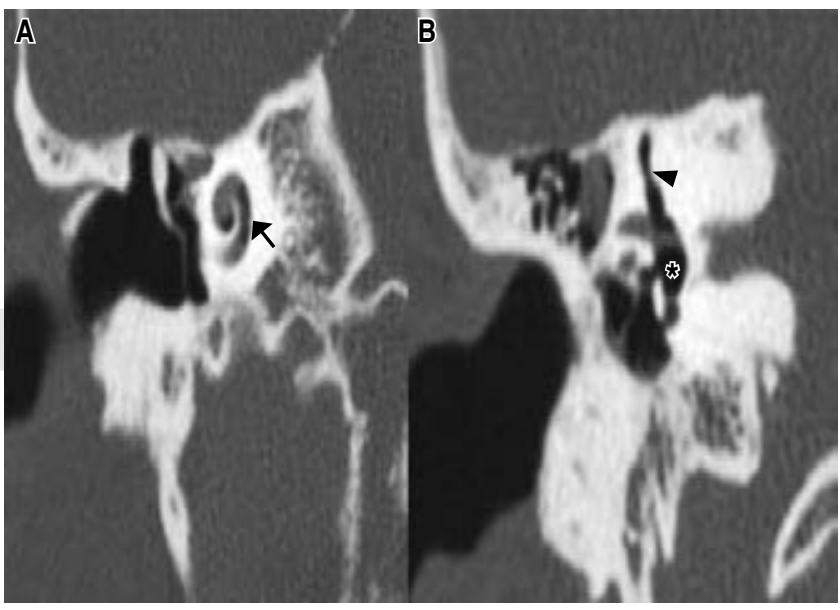

Figura 3: Tomografía computarizada de oído derecho en corte coronal. A) Neumolaberinto con extensión a y cóclea (flecha). B) Neumolaberinto con extensión a vestíbulo ('16) canal semicircular superior (cabeza de flecha). 


\section{DISCUSIÓN}

Presentamos un caso de fístula perilinfática con neumolaberinto como complicación secundaria a la erosión de la platina del estribo por colesteatoma. La fístula laberíntica es una de las complicaciones más comunes de la OMCC, a diferencia de la FPL, que es poco reportada en la literatura dentro del contexto de OMCC. Sheehy y Brackman reportaron una serie de 97 casos de fístula laberíntica; de éstos, más de $50 \%$ tenían el antecedente de OMC. El canal semicircular lateral es el sitio de afección más frecuente, por encontrarse más expuesto que el resto del laberinto. En este caso, nos llama la atención la afección predominante hacia la zona de la ventana oval, sin presentar afección del canal semicircular lateral, así como el inicio del cuadro súbito con vértigo intenso posterior a la limpieza en el consultorio. La presencia de vértigo sí se reporta en la mayoría de las series. McBee, en una serie de 79 casos, encontró una prevalencia de síntomas vestibulares de $90 \% .^{9}$

Debido al antecedente de OMCC y a la presentación clínica, consideramos que el colesteatoma erosionó la cadena osicular y la zona de la ventana oval, dejando el vestíbulo recubierto únicamente por la matriz de colesteatoma, la cual se removió durante la limpieza del oído medio, provocando así una FPL. En el contexto de OMCC con fístula laberíntica, existe controversia en cuanto al retiro de la matriz que recubre el sitio de la fístula. Al retirarla, se consigue una disminución de la resorción ósea y del riesgo de laberintitis supurativa; sin embargo, el riesgo de agravar la hipoacusia es alto. ${ }^{10}$ La experiencia del cirujano es determinante en esta decisión, sobre todo en casos en los que se realizó una mastoidectomía radical que permitirá el control de la matriz de colesteatoma en el consultorio si se decidió no removerla.

La presencia de neumolaberinto es infrecuente y es aún más rara en asociación con colesteatoma. Choi HG publicó un estudio retrospectivo que incluyó a 175 pacientes con fractura del hueso temporal. De éstos, 14 pacientes (8\%) presentaron neumolaberinto, $54 \%$ neumolaberinto localizado en vestíbulo o canales semicirculares y $46 \%$ localizado, además, en la cóclea. ${ }^{10}$ En una serie de casos publicada por Lee y Makino se reportaron tres casos de neumolaberinto asociado con la presencia de colesteatoma. Los tres pacientes eran de edad avanzada, al igual que en este caso. Estos autores proponen como explicación la disminución del calibre del acueducto coclear relacionada con la edad, el cual ocasiona un menor tránsito de perilinfa a través del conducto. De esta forma, es más sencillo que el aire entre hacia el oído interno cuando existe una fístula. ${ }^{5}$ En nuestro caso, se corroboró por tomografía computarizada la presencia de neumolaberinto con extensión al vestíbulo, canales semicirculares y cóclea.

El manejo es controversial, debido a que en la mayoría de los casos el aire suele reabsorberse de manera espontánea y la fístula suele sellarse con reposo. Se recomienda iniciar con manejo conservador y reservar el tratamiento quirúrgico para casos con sintomatología vestibular severa, progresión de la hipoacusia o FPL activa. ${ }^{5,8}$ Este caso se manejó en inicio de manera conservadora con reposo, evitando la maniobra de Valsalva, elevación de la cabecera, esteroide sistémico, antibiótico y antivertiginosos con lo cual se obtuvo una mejoría parcial de la sintomatología vestibular, pero no una mejoría auditiva.,7

Ante la presencia de una FPL, existe riesgo de laberintitis supurativa y meningitis, el cual es aún más elevado en casos como el nuestro, debido a la presencia de tejido de colesteatoma adyacente al sitio de fístula, por ello fue que se decidió el manejo quirúrgico. Se realizó mastoidectomía con técnica retrógrada sin encontrar más tejido de queratina en antro mastoideo, por lo que se decidió realizar exposición del ático con antroexclusión y obliteración de la ventana oval con tejido conectivo. ${ }^{11}$ Diferentes materiales han sido utilizados para el cierre de la fístula, tales como tejido conectivo, grasa, pericondrio, fascia o músculo temporal. ${ }^{12}$

En cuanto al pronóstico, Choi y colaboradores reportan mejoría de la sintomatología vestibular en $92 \%$ de los pacientes, a diferencia de la audición, que no tuvo mejoría en $83 \%$ de éstos. ${ }^{10}$ Hidaka y colegas concluyen que la presencia de neumolaberinto limitado al órgano vestibular es un factor de buen pronóstico para la recuperación de la audición, en comparación con la extensión a la cóclea, ${ }^{13}$ lo mismo que sucedió con el paciente del presente caso.

\section{CONCLUSIÓN}

El neumolaberinto es una complicación poco frecuente de la OMCC. La ventana oval como sitio de FPL en este contexto es una zona poco reportada, sobre todo cuando existe integridad de estructuras más expuestas a la erosión como el CSCL. Existe controversia respecto al manejo de la fístula perilinfática y el neumolaberinto, cualquiera que sea su etiología; sin embargo, consideramos que, ante el potencial riesgo de infección, como resulta en casos de fístula por colesteatoma, es imperativo erradicar la enfermedad y prevenirle complicaciones al sistema nervioso central. 


\section{BIBLIOGRAFÍA}

1. Prisman E, Ramsden JD, Blaser S, Papsin B. Traumatic perilymphatic fistula with pneumolabyrinth: diagnosis and management. Laryngoscope. 2011; 121 (4): 856-859.

2. Gersdorff MC, Nouwen J, Decat M, Degols JC, Bosch P. Labyrinthine fistula after cholesteatomatous chronic otitis media. Am J Otol. 2000; 21 (1): 32-35.

3. Ghiasi S. Labyrinthine fistula in chronic otitis media with cholesteatoma. J Pak Med Assoc. 2011; 61 (4): 352-355.

4. Rosito LPS, Canali I, Teixeira A, Silva MN, Selaimen F, Costa SSD. Cholesteatoma labyrinthine fistula: prevalence and impact. Braz J Otorhinolaryngol. 2019; 85 (2): 222-227.

5. Lee K, Makino K. Four cases of pneumolabyrinth without trauma. Otol Japan. 2012; 22 (3): 259-265.

6. Lee EJ, Yang YS, Yoon YJ. Case of bilateral pneumolabyrinth presenting as sudden, bilateral deafness, without temporal bone fracture, after a fall. J Laryngol Otol. 2012; 126 (7): 717-720.

7. Grundfast KM, Bluestone CD. Sudden or fluctuating hearing loss and vertigo in children due to perilymph fistula. Ann Otol Rhinol Laryngol. 1978; 87: 761-771.
8. Sousa Menezes A, Ribeiro D, Miranda DA, Martins Pereira S. Perilymphatic fistula and pneumolabyrinth without temporal bone fracture: a rare entity. BMJ Case Rep. 2019; 12 (3). pii: e228457.

9. Quaranta N, Liuzzi C, Zizzi S, Dicorato A, Quaranta A. Surgical treatment of labyrinthine fistula in cholesteatoma surgery. Otolaryngol Head Neck Surg. 2009; 140 (3): 406-411.

10. Choi HG, Lee HJ, Lee JS, Kim DH, Hong SK, Park B et al. The rates and clinical characteristics of pneumolabyrinth in temporal bone fracture. Otol Neurotol. 2015; 36 (6): 1048-1053.

11. Manrique R, Sanhueza I, Manrique M. Attic exposureantrum exclusion as an on demand surgical technique for cholesteatoma. Acta Otorrinolaringol Esp. 2013; 64 (1): 22-30.

12. Lao WW, Niparko JK. Assessment of changes in cochlear function with pneumolabyrinth after middle ear trauma. Otol Neurotol. 2007; 28 (8): 1013-1017.

13. Hidaka H, Miyazaki M, Kawase T, Kobayashi T. Traumatic pneumolabyrinth: air location and hearing outcome. Otol Neurotol. 2012; 33 (2): 123-131. 